\title{
Toward Haptic Communication: Tactile Alphabets Based on Fingertip Skin Stretch
}

\author{
Yoren Gaffary, Ferran Argelaguet, Maud Marchal, Adrien Girard, Florian Gosselin, Mathieu Emily, \\ Anatole Lécuyer
}

\begin{abstract}
This paper studies the possibility to convey information using tactile stimulation on fingertips. We designed and evaluated three tactile alphabets which are rendered by stretching the skin of the index's fingertip: (1) a Morse-like alphabet, (2) a symbolic alphabet using two successive dashes, and (3) a display of Roman letters based on the Unistrokes alphabet. All three alphabets (26 letters each) were evaluated through a user study in terms of recognition rate, intuitiveness and learnability. Participants were able to perceive and recognize the letters with very good results $(80 \%-97 \%$ recognition rates). Taken together, our results pave the way to novel kinds of communication using tactile modality.
\end{abstract}

Index Terms-Communication, tactile alphabet, fingertip skin stretch

\section{INTRODUCTION}

The richest form of textual communication between human beings is achieved through language. This information is commonly conveyed using the visual modality as when reading a book. However, in some situations using vision or audio to access information is inappropriate. For example, the light or the sound implied by receiving a text message in a theater could annoy the other people around who are trying to enjoy the play. Retrieving a password at an ATM or reading information on the dashboard of a car while driving could also be unsafe. Besides, disabled people could be visually or auditory deprived. The haptic modality could thus provide an interesting alternative to convey information.

Creating a tactile alphabet is a complex task [1]. A famous example of tactile alphabet is the Braille alphabet. The Braille alphabet can convey messages using raised dots. However, Braille is not intuitive or easy to learn for people accustomed to the Roman alphabet. Besides, it requires an active exploration through the fingertip which is not always possible.

This paper aims to conceive novel tactile alphabets to convey information without requiring any active exploration from the user. These tactile alphabets could be used by sighted people, used to the Roman alphabet, in cases visual and auditory senses are overloaded or not available. Such alphabets have to provide good recognition rates, but they also have to be intuitive and easy to learn. For us, one source of inspiration has been a game played by children which consists of recognizing a letter drawn on your back

- Y. Gaffary, F. Argelaguet, A. Girard and A. Lécuyer are at Inria, Rennes, France

E-mail: firstname.lastname@inria.fr

- M. Marchal is at INSA/Irisa, Rennes, France

E-mail:maud.marchal@irisa.fr

- F. Gosselin is at CEA, LIST, Gif-sur-Yvette, France E-mail: florian.gosselin@cea.fr

- M.Emily is at Agrocampus Ouest, Rennes, France E-mail:mathieu.emily@agrocampus-ouest.fr

Manuscript received M DD, 2017; revised M DD, 2017. with a finger by another person. This game consists of conveying letters using skin stretch. Contrary to the communication of information using Braille, the stimulation is here active and the user is passive: the person who receives the stimulus is not required to move and it is the finger of the other person which moves on his or her back. Besides, Braille is based on location of the dots, while the proposed skin stretch feedback uses movements of a single-end effector. This also enables the design of tactile letters which are based on the Roman alphabet, which should be intuitive for sighted people. Fingertips are an interesting area to explore for information communication as we use this part of the body to perceive most objects. For example, we use our fingertips to interact with the dashboard of a car, and blind people perceive their environment with touch. Because of their high density of tactile receptors [19], fingertips are thus a promising location to convey information through the haptic modality.

In this work, we focus on the design of tactile alphabets, from the most abstract to the most figurative. Our main contribution is the design and evaluation of three tactile alphabets based on skin stretch and applied at the level of the fingertip. The remainder of this paper is structured as follows. We first describe existing works on tactile icons and alphabets. We next introduce the use of skin stretch for tactile communication. Following this, we present three tactile alphabets mapped to the Roman alphabet and based on different communication strategies (temporal, symbolic and spatial). Then, we report a user study to assess these alphabets in terms of recognition rate and learning. Finally, the paper ends with a general conclusion.

\section{Related work}

This section describes existing work on the communication using the tactile modality. First, it presents tactile icons, an easy to learn but limited solution to communicate information. Second, it focuses on the Braille alphabet, which is able to convey various types of information although it is 
not easy to understand, especially for seeing or untrained people. Then, it details Morse code, which can be conveyed by tactile stimulations, such as vibrations, but remains difficult to learn. Finally, we describe alternatives of other tactile alphabet.

\subsection{Tactile icons}

Elaborating a complete, tactile alphabet enabling a correct and fast communication of information is a complex task. An easier way to communicate information was introduced by Brewster [4] and corresponds to tactile icons. Tactile icons are a form of tactile output which display information using iconic representations. They are generally based on vibrotactile feedback. Tactile icons are able to display various information based on different vibrotactile parameters, such as frequency, duration or body location. They also enable the communication of information in less time and space than Braille.

However, tactile icons are generally limited to a specific context such as expressing affective states to enrich instant messaging [31], [3] or supporting collaborative turn-taking [8], [9]. More recent studies however, have aimed to propose large sets of distinguishable tactile icons based on temporal features, such as rhythm or stimulus duration [18], [37] or by combining multiple tactile actuators [29]. Nevertheless, tactile icons remain unable to reproduce the same potential of a language, that can be obtained with the Roman alphabet.

\subsection{Braille alphabet}

The Braille alphabet was invented in 1825 and remains commonly used by blind people. Each letter is represented in this alphabet as a $3 \times 2$ matrix of vertical pins which can be perceived with fingertips, through active exploration. This language is most effective with blind people as they often have a more accurate tactile sensitivity at fingertips than seeing people (spatial JND $=1.04 \mathrm{~mm}$ vs $1.46 \mathrm{~mm}$ [38]). This smaller JND could be explained as blind people use and train their tactile perception more than sighted people. A fMRI comparison of the brain activity of blind and seeing people demonstrated a brain plasticity for Braille learning [33]. Generally, the learning is more effective if always with the same specific finger [35]. This explains why blind people usually use their index to read Braille as it is one of the most sensitive fingers [13].

Devices were proposed to encode Braille using multiple fingertips [11] or fingers [26]. However, using a specific device to read Braille makes it difficult to deploy in practice. Mobile phones were shown to be efficient to communicate Braille using vibrations [30].

However, the Braille alphabet presents several drawbacks. It requires a long learning time, especially for people who lost sight as it is very different from the Roman alphabet. The Braille alphabet also requires an active exploration by the user which is not always possible.

\subsection{Morse code}

As opposed to Braille, Morse code displays several advantages. Invented in 1838 , it is still used nowadays to communicate information [32]. Morse code can be conveyed using various modalities. It can rely on vision with a light, or audio with a soundspeaker [5]. More recently, researchers proposed a vibrotactile output to communicate information using Morse code [36]. This enables deafblind people to access information using this code. Due to its simplicity, Morse code seems also well adapted for people with physical disabilities [23], [39].

However, the Morse code also displays several limitations. As Braille, it requires an extended period of learning [21]. This explains why several studies proposed various approaches to improve its learning speed, for example, during paradoxical sleep or hypnosis [25], [34]. However, Morse remains traditionally difficult to learn and vibrotactile stimulations used to communicate information might be uncomfortable and tiring.

\subsection{Alternative solutions}

While they are still in use nowadays due to their efficiency, both Braille and Morse code are limited in terms of usage and learnability. This explains why new kinds of tactile alphabets and associated devices have been proposed. For instance, some devices consist of gloves equipped with numerous vibrotactile motors to convey information using the Malossi or the Lorm alphabets [6], [16]. However, these gloves are thick and prevent the whole hand from being used for other possible tactile perception purposes. More recently, Liao et al. proposed a tactile wrist device based on four tactors arranged in a square [22]. Using a successive activation of the tactors, they were able to convey tactile letters with a spatial pattern as close as possible to the Roman alphabet.

Another promising way to convey information using haptic feedback is skin stretch. Pasquero et al. [27] proposed a tactile device to convey information using this kind of tactile feedback. Chouvardas et al. [10] proposed an overview of existing tactile displays. They highlighted that some tactile receptors such as Ruffini Endings were involved in the perception of skin stretch. Multiple studies highlighted the potential of skin stretch to convey information. Gleeson et al. [15] used this kind of stimulation to convey information about direction at the fingertip. Moreover, Gwilliam et al. [17] showed that skin stretch offers a great precision for interpreting directional information. This suggests that this kind of feedback might be relevant for efficiently discriminating tactile patterns based on changes of direction. Furthermore, skin stretch was also deployed on a steering wheel to convey information to a driver [28]. The proposed stimulation had only 1 degree of freedom as it consisted of a lateral movement, limiting the transmission of various information.

In the remainder of the paper, we aim to design novel tactile alphabets based on skin stretch. We notably designed a tactile version of the Morse code based on skin stretch, and two other tactile alphabets based on symbolic or simplified representations of the Roman alphabet.

\section{INTRODUCING SKIN STRETCH FOR TACTILE COMMUNICATION}

This section introduces the use of skin stretch on fingertips in order to support tactile communication. First, we present 
and describe the device we used for enabling such specific kind of tactile stimulation. Second, a feasibility study investigates the use of such tactile feedback to communicate information using tactile patterns. This study also aims to highlight clues on the design of future tactile alphabets.

\subsection{Concept}

In this paper, we propose to use a skin stretch feedback with 2 degrees of freedom (DoF). More specifically, we aim to stimulate fingertips as they are one of the most sensitive part of the body. To achieve this objective, we use a tactile device called "HapTip" [14]. This device stimulates the fingertip using a tactor corresponding to a plastic cap moving in the horizontal plane thanks to two miniature DC motors (see Figure 1). The device is designed to be both compact and light. Its position can be adjusted to the participant's dominant index as it corresponds to the most effective finger to discriminate tactile stimuli [13]. It uses cable capstan reducers with pulleys mounted on ball bearings and backlashfree plain bearings in the joints to avoid both backslash and friction and to provide a continuous movement. The position of the device is computed using miniature Hall effect sensors at the joint level (sensors are located in the driven pulleys). It is controlled through the manufacturer API at $333 \mathrm{~Hz}$, by sending every $3 \mathrm{~ms}$ to the device the 2D position it has to reach. The device can be portable or fixed to a table.

The technical specifications of the device are reported in Table 1 . In order to ensure that the device is sufficiently precise to implement the subtle displacements required for tactile communication, we further investigated the positional accuracy of the tactor. During the device's development process, its kinematic was identified and calibrated by moving the tactor with a precision 2D laboratory positioning table while recording both the sensors' responses and the joints' angles (computed from the device's CAD model). Results are given in Figure 2, left. A linear regression was used to get a simple model for each joint. It is worth noting that, by moving the end effector and registering the sensors response at the joint level, this procedure ensures capturing all nonidealities occurring between the joints and the tactor (e.g. sensors placement errors, sensors' non-linearity, links' size errors). Also, the use of miniature cable reducers prevents backlash between the actuators and joints. Thanks to our design, these no-load characteristics are almost preserved in the presence of the finger. Indeed, implementing the position control in closed loop at the joint level allows minimizing the influence of the compliance-induced cable deformations on the positioning accuracy. Also, using short links and backlash free bearings minimizes structure backlash and deformation under load. Finally, having a force capacity able to deform the skin a few millimeters minimizes the influence of the finger resistance to movements. As can be seen in the picture, the model fits well experimental data on joint 1 (angle q1), with errors below $3.5^{\circ}$ all over the workspace (mean $0.77^{\circ}$ ), but larger errors are obtained on axis 2 (angle q2), up to $5.3^{\circ}$ (mean $1.98^{\circ}$ ). This results in small errors when computing the moving cap position from the sensors' voltages. These errors can be estimated using the geometrical model of the robot. Results are given in
Figure 2, right. Mean error over the workspace is $0.2 \mathrm{~mm}$. The range of movements of our tactile alphabets being between $2.82 \mathrm{~mm}$ and $4 \mathrm{~mm}$ (see next sections for details), we can thus conclude that the device's accuracy is sufficient for our experiments.
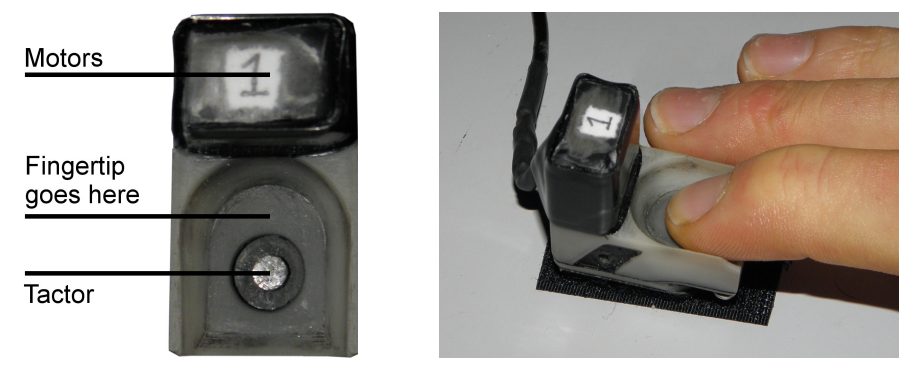

Fig. 1: Close-up view of the HapTip used in our study.

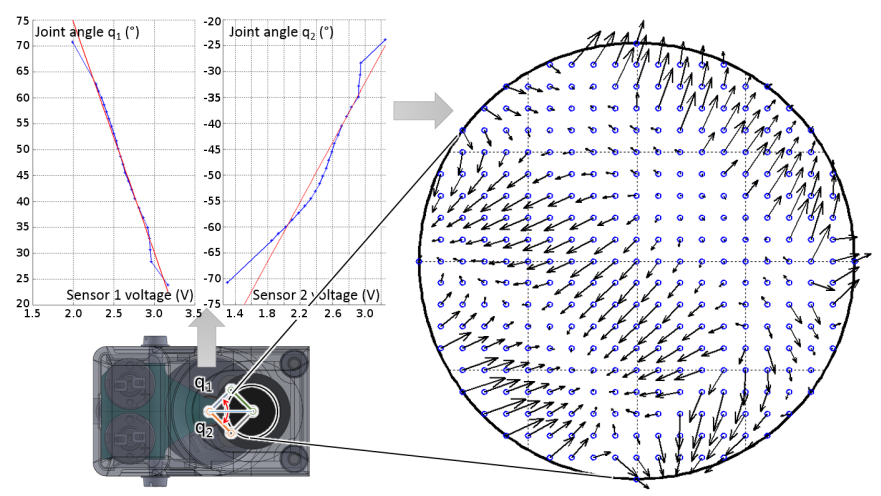

Fig. 2: Haptip internal structure and joint angles as a function of the sensors response (left, blue: experimental data, red: linear regression model) and positional accuracy over the workspace (right, blue dots: desired positions, black arrows: deviation from setpoint positions. A square corresponds to $1 \mathrm{~mm}$ ).

TABLE 1: Main characteristics of the HapTip.

\begin{tabular}{|l|l|}
\hline Dimension $(\mathrm{W} \times \mathrm{H} \times \mathrm{D})$ & $20.4 \mathrm{~mm} \times 35 \mathrm{~mm} \times 34.1 \mathrm{~mm}$ \\
\hline Weight & $22 \mathrm{~g}$ \\
\hline Degrees of freedom & 2 \\
\hline Motion range & $\pm 2 \mathrm{~mm}$ in any direction \\
\hline Mean position accuracy & $\pm 0.2 \mathrm{~mm}$ in any direction \\
\hline Force & $3.4 \mathrm{~N}$ in any direction \\
\hline Max speed & $150 \mathrm{~mm} \cdot \mathrm{s}^{-1}$ \\
\hline
\end{tabular}

\subsection{Feasibility study}

We conducted a feasibility study on the potential of skin stretch to convey information at fingertips. This study focused on the recognition rate of tactile patterns using different tactile rendering. According to the just noticeable difference for spacial resolution of the fingertip tactile perception (1.46 $\mathrm{mm}$ [38]), we created tactile patterns presenting a fixed number of 4 spots. Six different patterns were designed, each corresponding to a possible traveling order for the 4 


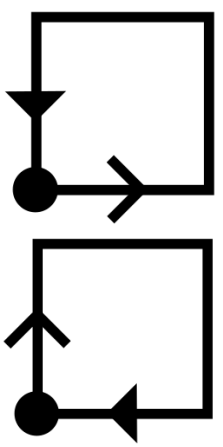

(a) Squares

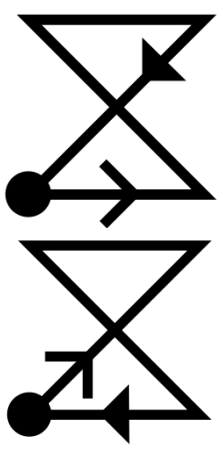

(b) Vertical hourglasses

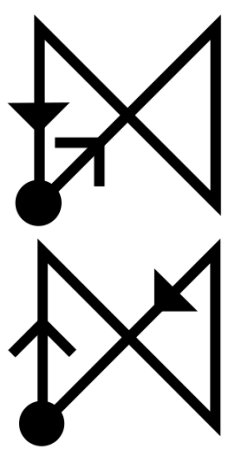
hourglasses (c) Horizontal

Fig. 3: Tactile patterns presented to participants in our feasibility study. The black dot corresponds to the starting and ending position of the tactor of the device. Arrows represent the traveling direction of the tactor.

spots. These patterns corresponded to two squares, two vertical hourglasses and two horizontal hourglasses, illustrated in Figure 3.

Two tactile rendering techniques were also tested. The first one is a continuous rendering technique (CT), in which the pattern is displayed by the tactor at a constant speed. The second rendering technique is an inflexion points-based rendering technique (IT), in which the tactor makes a break at each spot. In this second technique, the tactor stopped moving each time it was supposed to change direction. All stimuli lasted one second, and the tactor moved at constant speed. The position loop update rate of the tactor was set to $333 \mathrm{~Hz}$. In the (IT) rendering technique, breaks lasted the same duration as one dash of the pattern.

14 participants aged from 20 to $30(\mathrm{M}=23, \mathrm{SD}=2.52)$ were involved in this feasibility study. The six patterns were presented in a random order, 15 times each, to participants. All participants completed the experiment with the two rendering techniques presented sequentially in a counterbalanced way. The experiment lasted around 30 minutes.

Results showed that for all patterns and rendering techniques, the recognition rates were above chance level $(\mathrm{M}$ $=54.6 \% \gg 16.7 \%=1 / 6)$. The lowest recognition rate concerned the hourglasses which were sometimes mistaken for each other $(50.1 \%$ mean correct recognition rate for the hourglasses, $63.7 \%$ for the squares). We observed that the squares were better recognized when using the (CT) rendering than when using (IT) (CT: $66.2 \%$ vs IT: $61.2 \%$ ). However, a paired Wilcoxon signed-rank test showed that this difference was not significant $(p=0.14$, Qobs $=202$ ). However, hourglasses seem to be better recognized with (IT) (IT: $52.6 \%$ VS CT: $47.7 \%$ ). This result was significant $(p=0.025$, Qobs $=370)$. Concerning the strategies used to identify the tactile patterns, 7 participants reported they were mostly focused on the first and last segments of the tactor motion to identify the pattern, i.e., on only two dashes.

In a nutshell, our results were very encouraging in terms of recognition rate, with an average performance close to $55 \%$, being above chance level (17\%). Adding breaks at inflexion points seems to improve the recognition rates of patterns presenting shapes that resemble each other more. Besides, half of the participants reported that they based their pattern recognition on the first and last perceived segments. This implies that the other segments of the patterns were not always necessary and that two dashes could be sufficient to obtain good recognition rates.

\section{DESIGN OF THREE NOVEL TACTILE ALPHABETS BASED ON SKIN STRETCH}

Encouraged by the results of our feasibility study, we designed three novel tactile alphabets using skin stretch on the fingertips. The letters of these alphabets are mapped to the letters of the Roman alphabet: each alphabet contains twenty-six different letters (see Table 2). These tactile alphabets are based on different communication strategies, presented hereafter from the most abstract to the most figurative alphabet:

- Our first tactile alphabet communicates letters using a temporal representation. This alphabet is based on Morse code and corresponds to our ground truth.

- Our second alphabet communicates letters using symbolic spatial patterns. This alphabet called "TwoDashes" was designed taking into account the guidelines derived from our feasibility study.

- Our third alphabet communicates letters by drawing a simplified, spatial version of their shapes. This alphabet corresponds to a tactile version of the existing Unistrokes alphabet. This alphabet is the closest to the Roman alphabet.

The following sections detail successively the design of the letters of these three tactile alphabets. The video attached to the paper presents the rendering of the three tactile alphabets on the Haptip device.

\subsection{Tactile alphabet based on Morse code}

Some alphabets, like Morse code, use temporal signals to convey information. As Morse code is widely spread and very efficient, we used this tactile alphabet as ground truth. Our version of Morse-based alphabet relies on short and long tactile signals, varying only in their duration. Each signal is separated from another by a short break. Table 2 represents the coding of each Roman letter in Morse code.

We adapted the Morse code to the HapTip since we are aiming to convey information using skin stretch on fingertips. While vibrations are sufficient for Morse code, using skin stretch for all proposed alphabets enables us to compare their efficiency. Each signal is displayed using a specific displacement of the tactor. A signal is represented by a forth/back movement of the tactor from the top to the bottom, then to the top again (see Figure 4). We used the vertical axis of movement as it is the best perceived axis with fingertips [12], [13]. The main difference between short and long signals concerns their duration. A short signal is represented by a vertical movement of the tactor lasting $0.3 \mathrm{~s}$. Then, the duration of long signals (respectively, breaks) was set to $0.9 \mathrm{~s}$ (respectively, $0.3 \mathrm{~s}$ ). 
TABLE 2: Our three tactile alphabets based on skin stretch. For Morse, short and long tactile movements of the tactor are represented respectively by dots and dashes. For TwoDashes, dashed lines correspond to diagonals, displayed with slower displacement of the tactor than vertical and horizontal lines. For Unistrokes, hollow circles represent inflexion points where the tactor makes a break.

\begin{tabular}{|c|c|c|c|}
\hline Letter & Morse & TwoDashes & Unistrokes \\
\hline A & • - & -1 & $\uparrow$ \\
\hline B & - $\cdots$ & $\leftrightarrows$ & $>$ \\
\hline C & 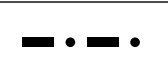 & $\vec{\square}$ & 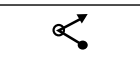 \\
\hline $\mathrm{D}$ & - •. & $\vec{\vartheta}$ & $<$ \\
\hline $\mathrm{E}$ & . & $\rightleftarrows$ & $\hookleftarrow$ \\
\hline$F$ & •・日・ & $\Gamma$ & $q$ \\
\hline G & $-\sigma$ & $\therefore$ &.$\oint$ \\
\hline $\mathrm{H}$ & $\cdots \cdot$ & ᄂ. & $q$ \\
\hline I & •. & d & $i$ \\
\hline $\mathrm{J}$ & $\cdot-\pi$ & 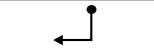 & $\stackrel{d}{ }$ \\
\hline K & $-\cdot-$ & 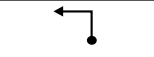 & $\nearrow$ \\
\hline L & •-・・ & $\stackrel{L}{\longrightarrow}$ & $\stackrel{d}{\leftrightarrow}$ \\
\hline M & -- & I. & $\Omega$ \\
\hline $\mathrm{N}$ & - & $d x$ & $\Omega$ \\
\hline $\mathrm{O}$ & 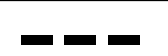 &.$\uparrow$ & 8 \\
\hline $\mathrm{P}$ & •- - & 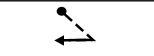 & x \\
\hline Q & $-\sigma \cdot-$ & $\stackrel{\leftrightarrow}{\hookrightarrow}$ & $\mathcal{\alpha}$ \\
\hline $\mathrm{R}$ & • - . & 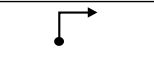 & $\searrow$ \\
\hline S & $\bullet \cdot \bullet$ & $<-1$ & $\stackrel{\square}{\square}$ \\
\hline $\mathrm{T}$ & - & $\neg$ & $\leftrightarrow$ \\
\hline U & ..- & $l^{\prime}$ & $\gamma$ \\
\hline V & •・・ & 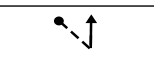 & $v$ \\
\hline W & •- - & t, & $\mathscr{b}$ \\
\hline$x$ & - & $\because$ & $\gamma$ \\
\hline Y & 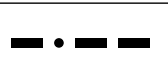 & $x y$ & $\zeta$ \\
\hline Z & - • • & 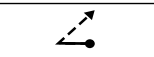 & $\stackrel{2}{\longrightarrow}$ \\
\hline
\end{tabular}

\subsection{Tactile alphabet based on TwoDashes}

Our second alphabet was designed based on the results of our feasibility study. We considered two guidelines to be used in the design of this alphabet. The first one was that the alphabet should rely on the same four different spots of the tactor used in our feasibility study: top-left, topright, bottom-left and bottom-right corners. All letters of the alphabet should be described as an ordered list of tactor displacements from one spot to the next one.

Second, half participants in the feasibility study reported they used only the first and last displacements of the tactor

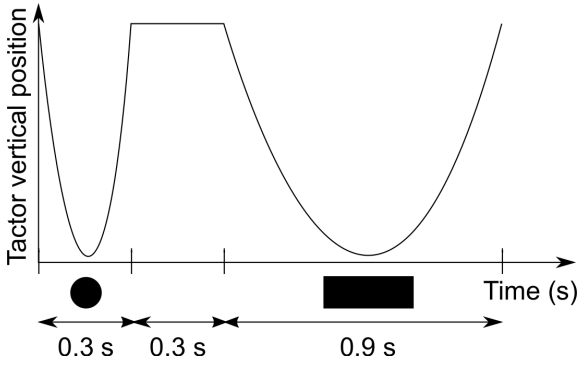

Fig. 4: Representation of the vertical movement of the tactor corresponding to the letter ' $\mathrm{A}$ ' in Morse code with the HapTip. The duration of the movement indicates if it is a short or a long signal.

to identify a pattern, and that it was difficult to remember the other displacements between them. Thus, we proposed to use only two dashes to represent each letter of the alphabet. Using exactly two dashes also has the advantage that participants know in advance the number of dashes composing each letter.

Each letter of the TwoDashes alphabet is composed of 4 possible starting spots, crossing 3 possible intermediate points and ending at 2 possible spots, providing $4 \times 3 \times 2=$ 24 possible patterns. As we require to convey 26 patterns to represent the 26 letters of the Roman alphabet, we arbitrarily added one horizontal and one vertical tactor displacement with their starting point corresponding to the ending point. We mapped the letters of the TwoDashes alphabet with those of the Roman alphabet to match them as close as possible. For instance, the 'L' letter corresponds to a down, then right skin stretch, whereas the letter ' $\mathrm{I}$ ' corresponds to a vertical skin stretch. As observed in literature, the perception of a $45^{\circ}$ diagonal using skin stretch could be confused with horizontal and vertical displacements [13]. This implies that two letters could be difficult to discriminate when two different dashes differ by only $45^{\circ}$ degrees. To help users distinguishing diagonal from non-diagonal tactor displacements, we propose to change the speed of diagonal displacements.

We did some informal tests to experimentally set the speed of the tactor during vertical, horizontal and diagonal dashes. The speed of diagonal (respectively, non-diagonal) displacements of the tactor was set to $6 \mathrm{~mm} \cdot \mathrm{s}^{-1}$ (respectively, $3 \mathrm{~mm} \cdot \mathrm{s}^{-1}$ ). We did not need to use inflexion points, as there are always two dashes with different directions for each letter. The final design of the letters of TwoDashes alphabet is illustrated Table 2.

TABLE 3: Properties of our three tactile alphabets, mean and standard deviation are reported (M (SD)).

\begin{tabular}{|l|l|l|l|}
\cline { 2 - 4 } \multicolumn{1}{c|}{} & Morse & TwoDashes & Unistrokes \\
\hline Duration (s) & $2.5(0.9)$ & $2.2(0.6)$ & $2.9(1.5)$ \\
\hline $\begin{array}{l}\text { Traveled distance } \\
(\mathrm{mm})\end{array}$ & $12.6(3.8)$ & $6.4(0.6)$ & $6.4(2.5)$ \\
\hline Bitrate (letter/min) & 24.3 & 27.9 & 20.9 \\
\hline
\end{tabular}




\subsection{Tactile alphabet based on Unistrokes}

The main idea of this third alphabet is to directly draw the spatial representation of the Roman alphabet on the fingertips using skin stretch. We based our work on a continuous representation of the Roman alphabet called Unistrokes that represents the Roman letters using 2 DoF [7]. Unistrokes is an efficient alternative and more simplistic version than Graffiti [24]. Table 2 represents the coding of each Roman letter with the Unistrokes alphabet. Thus, we propose to mimic the letters of the Unistrokes alphabet using skin stretch. As suggested in our feasibility study, we set breaks at inflexion points (see Table 2). We performed pilot studies to set the speed of the tactor and duration of breaks. The mean speed of the tactor was set to $5 \mathrm{~mm} . \mathrm{s}^{-1}$. At inflexion points, the duration of breaks was set to $0.4 \mathrm{~s}$.

Table 3 summarizes the duration, the traveled distance and the bitrate related to the presentation of the letters with the three different alphabets.

\section{USER STUDY}

We conducted a user study to assess the recognition rate and the learnability of our three tactile alphabets. As in the children's game mentioned in introduction, participants were asked to report the letters they perceived using skin stretch on their fingertips. We measured the recognition rate and recognition time for each presented letter. We supposed that the Morse-based alphabet would show the best recognition rates, but that the two other alphabets would perform rapidly better across sessions. More precisely, we supposed that the closer to Roman letters, the better the alphabet would be easy to learn. The experiment was completed by the participants in five different sessions spread on five different days.

\subsection{Apparatus}

The experimental setup was based on the same HapTip tactile device as described in our feasibility study. The placement of the device on the table was set according to the length of the participant's finger. The device was fixed on the table in front of the dominant hand of the participant using velcro which prevented the device from moving during the experiment. The participants were seated with their forearms placed on a table (see Figure 5). They were in front of a 24 -inch screen. A keyboard was placed in front of the non-dominant hand of participants.

\subsection{Participants}

Nine participants ( 2 females) took part in the experiment, aged from 23 to 40 (mean $=28, \mathrm{SD}=5.2$ ). All participants identified their right hand as the dominant one and were proficient with the Roman alphabet. Regarding the knowledge of the tested alphabets, half of the participants reported to have basic knowledge of the Morse alphabet, and no participant had knowledge of the Unistrokes or the TwoDashes alphabets.

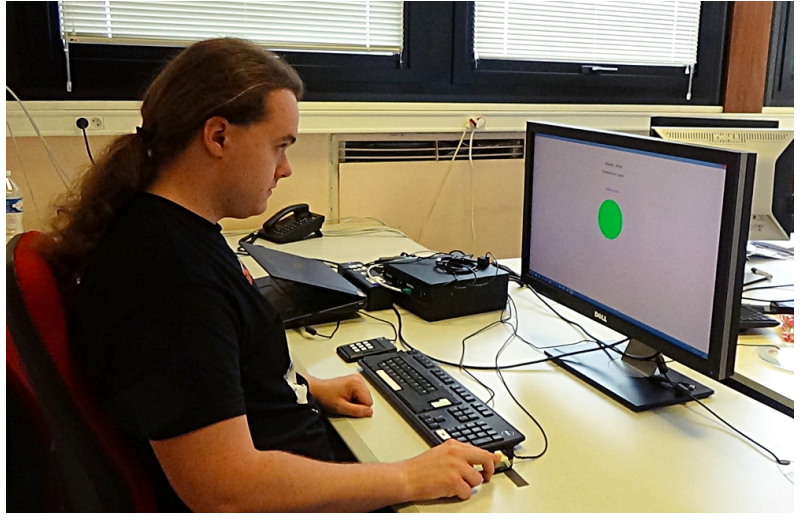

Fig. 5: Experimental setup. A participant perceives a letter using skin stretch at his index, and enters his choice (letter) using the keyboard with his non-dominant hand.

\subsection{Experimental design}

\subsubsection{Procedure}

The experiment was divided into three blocks completed successively, each one corresponding to one tactile alphabet. For each block, each letter of the alphabet was presented once, in a random order. The goal of the user was to recognize the letter which was rendered using the HapTip on the index finger of their dominant hand. Each block started with a short training session, in which participants had to recognize sequentially four random letters. For each tested letter there were two steps: 1) the letter is rendered using the HapTip and 2) participants had to press the key from the keyboard which matched the recognized Roman letter. During the second step, the mapping between the tactile alphabets and the Roman alphabet was displayed visually on the screen. Participants were not informed if they selected the correct letter. Before each stimulus, the tactor performed at high speed at least a complete circle to reach the starting position of the next stimulus. This was intended to prevent the user from feeling where it stopped, giving an additional cue on the stimulus displayed.

Between each block, participants filled a subjective questionnaire gathering their impressions about their performance. In order to analyze the user adaptation to the different tactile alphabets and their learning curves, the experiment was repeated in 5 different sessions, each one lasting 30 minutes. The sessions were scheduled over different days, over 1 week.

\subsubsection{Conditions}

Three conditions were evaluated in this experiment. The first one corresponded to the tactile ALPHABET presented: MORSE, UNISTROKES or TWODASHES. All participants had to recognize all letters from these three alphabets. The second condition was the LETTER itself. Finally, the SESSION number was also considered as an independent variable to evaluate the evolution of association time and learning of the tactile letters through time.

\subsubsection{Collected data}

For each presented letter, we collected 2 objective measures: 
Mo1 Recognized letter. The Roman letter recognized by the participant.

Mo2 Letter association time. The time between the display of the alphabet on the screen and the selection of a letter on the keyboard.

At the end of each of the five sessions, the following questions were asked to participants for each presented alphabet. They were answered using a 5-Likert scale, 1 meaning the lowest score, and 5 meaning the highest score:

Ms1 Intuitivity of the alphabet.

Ms2 Improvement through the session.

Ms3 Improvement compared to the last session. This question was not asked after the first session.

At the end of the last session, two additional questions were asked to participants for each presented alphabet.

Ms4 How do you evaluate the easiness of remembering the tactile signal, from the moment you perceived it to the moment you associated a letter to it?

Ms5 Through the sessions, how do you evaluate the easiness of learning the alphabet, i.e., the association between the tactile and the roman letters?

\subsection{Results and Analysis}

\subsubsection{Objective results}

Recognition rate (Mo1) - To study the recognition rate of the letters as a function of the alphabet and the session, we used a logistic regression model on the collected data to model the probability of recognition with respect to the independent variables ALPHABET, LETTER and SESSION defined in the experimental design. The participants were considered as a random effect in the model. We performed an analysis of deviance of the logistic regression model and we found a significant marginal effect for ALPHABET $(p<0.001)$, LETTER $(p<0.001)$ and SESSION $(p<0.001)$. We performed a post-hoc analysis on the SESSION condition to study if we had a training effect. We used a Tukey test adapted to the logistic generalized regression model and we found that the first session has significantly lower recognition rates than the other sessions $\left(Z_{2}=-4.06, Z_{3}=-3.76, Z_{4}=-5.18\right.$, $Z_{5}=-5.94$ for sessions 2 to 5 , and $p<0.001$ for sessions 2,4 and $5, p=0.002$ for session 3 ). We therefore removed data of session 1 from the data set (see also Figure 6a for the evolution of the recognition rate of the different alphabets in function of the sessions). We then performed again an analysis of deviance of the logistic regression model without the SESSION condition and we found a significant marginal effect for both ALPHABET $(p<0.001)$ and LETTER $(p=0.004)$, as well as an interaction effect between ALPHABET and LETTER $(p<0.001)$. We performed a post-hoc analysis on the ALPHABET using a Tukey test and we found that the MORSE Alphabet (0.97) was significantly better recognized than the other alphabets $(Z=12.35, Z=10.03$ for the TWODASHES and UNISTROKES alphabets respectively, $p<0.001$ for both). We also found that the UNISTROKES alphabet $(0.80)$ was significantly better recognized than the TWODASHES alphabet $(0.73)(Z=3.96, p<0.001)$.
Association time (Mo2) - We conducted a two-way ANOVA on SESSION and ALPHABET concerning the association time (see Figure $6 \mathrm{~b}$ ). We observed main effect of the ALPHABET $\left(F_{2,16}=13.67, p<0.001, \eta_{p}^{2} \leq 0.63\right)$. The TwODASHES alphabet had significantly highest association time (5.63 s). There was no difference between the MORSE (4.41 s) and the UNISTROKES alphabets $(3.99 \mathrm{~s})$. We also observed a main effect of the session $\left(F_{4,32}=24.86, p<0.001, \eta_{p}^{2} \leq 0.76\right)$. The association time for the first session was significantly higher than the other sessions. The association time for the second session was higher compared to the last one. There was no significant difference between the association times for sessions 3,4 and 5 . The interaction effect showed that the association time decreased faster for the UNISTROKES alphabet, especially for the first and second sessions $\left(F_{8,64}=4.17\right.$, $p<0.001, \eta_{p}^{2} \leq 0.34$ ).

Confusions - Some letters were mistaken with another specific letter more than $10 \%$ of the time. Table 4 illustrates the main confusions between letters for the differ-

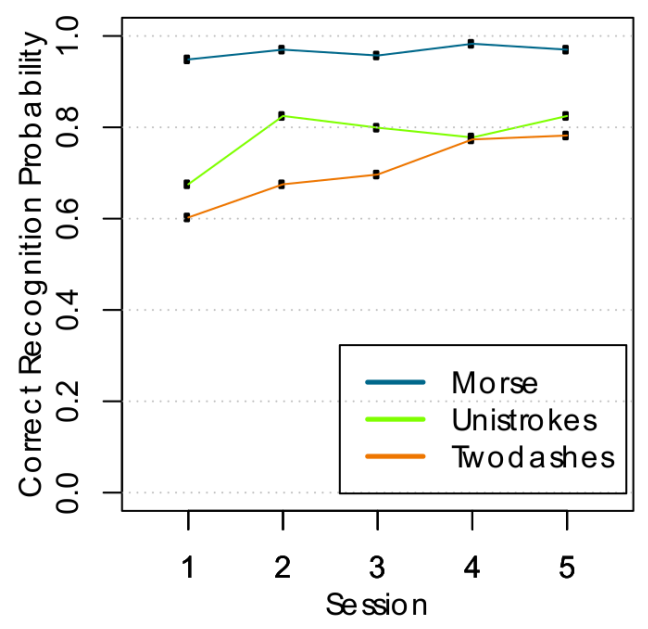

(a) Probability of correct recognition (Mo1) depending on the alphabet and the session number.

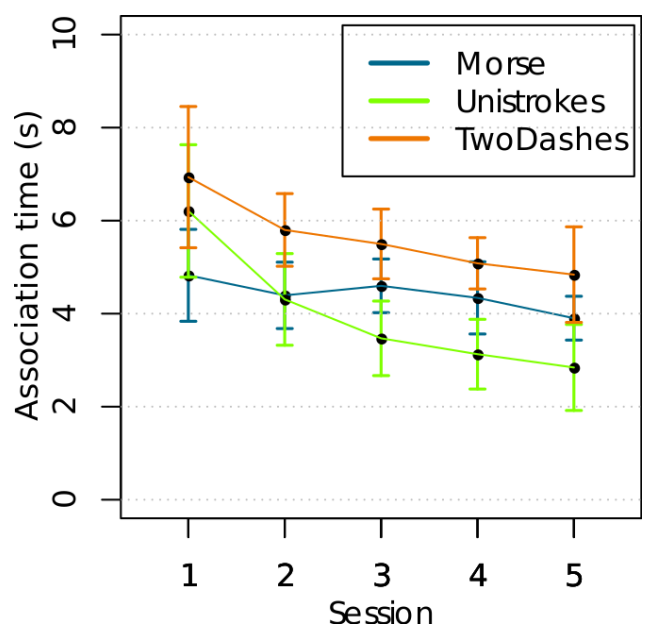

(b) Mean letter association time (Mo2) depending on the alphabet and the session number (CI $95 \%$ ).

Fig. 6: Probability of correct recognition and association time. These plots display the results for the three tactile alphabet depending on the session number. 
ent tactile alphabets. No confusion was reported for the Morse. Unistrokes and TwoDashes reported different kinds of confusion. For Unistrokes, it concerns letters for which the pattern is similar, but rotated from $45^{\circ}$. For TwoDashes, it concerns letters for which one dash of the pattern differs by $45^{\circ}$ to the other one.

\subsubsection{Subjective results}

Participants evaluated that the Unistrokes alphabet was more intuitive (Ms1, M = 2.71) compared to Morse and TwoDashes (1.78 and 1.91). A Friedman rank sum test showed that this result was significant $p=0.01$.

Participants evaluated they improved the more during sessions with the Unistrokes alphabet $(\mathbf{M s 2}, \mathbf{M}=2.51)$ compared to Morse and TwoDashes (2.13 and 2.29, $p=0.044)$.

Participants evaluated they improved the more between sessions with the Unistrokes alphabet (Ms3, $\mathrm{M}=2.56)$ compared to Morse and TwoDashes (2.00 and 2.39, $p=0.018)$.

Participants reported no difference between the three alphabets in terms of easiness of remembering the tactile signal (Ms4). However, they evaluated Unistrokes to be easier to learn $(\mathbf{M s 5}, \mathbf{M}=3.44)$ than Morse and TwoDashes (2.22 and 1.88, $p=0.012)$.

\subsection{Discussion}

After five sessions, the three proposed tactile alphabets displayed high recognition rates (from $80 \%$ to $97 \%$, with a chance level of only $3.8 \%$ ). This indicates that all our tactile alphabets are effective to convey information successfully.

The three investigated tactile alphabets showed complementary advantages. Concerning recognition rate, TwoDashes and Unistrokes displayed less efficiency than Morse which presented nearly perfect recognition rate, although none of our participants declared a great expertise on this alphabet. This may imply that the temporal cues of Morse code were easier to recognize than the spatial symbols of TwoDashes and Unistrokes. However, recognition rates improved over sessions for these two alphabets. This suggests that the association between their letters and the letters of the Roman alphabet is easy to learn. However, Unistrokes seems easier to learn than TwoDashes as its recognition rate significantly increased from the second session.

We also investigated the recognition confusions for Unistrokes and Twodashes to identify which letters could be easily confused. The low amount of confusions in TwoDashes suggests that simple, symbolic patterns are sufficiently effective to convey letters with little ambiguity. But Unistrokes highlighted that two patterns differing only by a rotation of $45^{\circ}$ could be easily confused. This suggests that an efficient tactile alphabet using skin stretch on a surface as small as a fingertip should keep a balance between the simplicity of the patterns of the tactile letters and its closeness to the Roman alphabet.

The proposed approach using skin stretch displays some practical advantages compared to other devices. For example, the UniBraille device proposed by [26] was designed to convey letters encoded with the Braille alphabet using tactile stimulations on fingers. Each stimulus needed to last at least $2 \mathrm{~s}$ to obtain an recognition rate of $88.57 \%$, which is similar to the presentation time of the alphabets proposed in this study. However the encoding of the alphabet requires three fingers on both hands to convey each letter. The three alphabets proposed in the current study display the advantage of requiring only one finger, which is less intrusive. The alphabet also remains less intuitive for sighted users as the representation of the letters is not similar to the Roman alphabet.

The type of stimulation proposed by our device displays common points with EdgeVib, described by Liao et al [22]. This device uses four different tactors located on the wrist to convey the letters of the Unistrokes alphabet. Their study proposes an interesting solution to discriminate letters that have a similar representation, at the cost of increasing the complexity of the tactile patterns. Yet, the proposed stimulation is limited to only four possible stimuli locations, depending on the location of the tactors. Our proposed approach using skin stretch is based on a tactor which can move in $2 \mathrm{D}$, which enables us to perform stimulations including displacements. The Haptip device has not been previously evaluated in terms of rendering capacities and non-idealities (i.e., actual vs. expected tactor displacement). But even if some non-idealities would have been present, our results are still very promising in terms of recognition rate, which is very encouraging for future work.

The displacement speed of the tactor could be used to convey more stimuli parameters, or to let the user decide his or her own preference for the reading speed.

A long-term perspective would be to investigate the recognition of words or even sentences, extending our approach to "tactile languages". Our current work is of course preliminary and much research remains to be done. It implies notably to define how transitions between letters and words are rendered using tactile feedback. It also induces numerous user studies aiming at fully characterizing the error and transfer rates in such case of more complex content.

More generally, our study has focused on recognition rates and association times for our proposed alphabets, and future work could investigate the required time to learn the different alphabets. Moreover, future work could also study the difference in learning and in recognition rate between sighted people and recently-blind people who could be familiar with the Roman alphabet. Besides, our study focused on fingertip stimulation, in a context where the user is not interacting with objects with his/her fingers. It could also be interesting to study how skin stretch could be applied to other areas of the body to convey information to the user. The resolution of the tactile perception would probably be less effective at other body areas but this would enable freeing the hands of the user and let him/her interact with other devices at the same time.

Future studies could explore more in-depth the tactile resolution of the fingertips. Taking even more into account the tactile perception of fingers from a neurophysiological point of view could enable dealing with fingertips' tactile resolution and refining the design of our tactile alphabets and their associated tactile devices. References such as [19], [20], [2] could serve as a good starting point for such studies. 
TABLE 4: Recognition confusions. This table reports an example of pairs of presented/recognized symbols where the presented symbol is mistaken with the other symbol at least $10 \%$ of the time.

\begin{tabular}{|c|c|c|c|c|c|}
\hline Alphabet & Confusion type & $\begin{array}{l}\text { Presented } \\
\text { letter }\end{array}$ & $\begin{array}{l}\text { Selected let- } \\
\text { ter }\end{array}$ & $\begin{array}{l}\text { Number of concerned } \\
\text { pairs of letters }\end{array}$ & $\begin{array}{l}\text { Mean percentage of } \\
\text { confusion }\end{array}$ \\
\hline Unistrokes & Rotation of $45^{\circ}$ & $\uparrow$ & $\pi$ & 16 & 16.7 \\
\hline Unistrokes & Vertical symmetry & $\stackrel{\leftrightarrow}{\leftrightarrows}$ & $\ddot{M}$ & 2 & 14.5 \\
\hline TwoDashes & $\begin{array}{l}\text { One of the two dashes differs by } \\
45^{\circ}\end{array}$ & $a \cdot \hat{\jmath}$ &.$\uparrow$ & 7 & 13 \\
\hline TwoDashes & Rotation of $45^{\circ}$ & $x y$ & $\uparrow \cdot$ & 1 & 20 \\
\hline TwoDashes & $\begin{array}{l}\text { The } L \text { is mistaken with the last } \\
\text { part of a C }\end{array}$ & $\stackrel{L}{\longrightarrow}$ & $\vec{y}$ & 1 & 18 \\
\hline
\end{tabular}

\section{CONCLUSION}

This paper aimed to introduce tactile communication based on skin stretch. Skin stretch has the advantage to free visual and auditory senses, which could be crucial in some contexts as driving. It also enables richer stimulations than vibrations as it includes a temporal and a spatial component and does not require movements from the user. We designed and evaluated three novel tactile alphabets using skin stretch: Morse, TwoDashes and Unistrokes. These tactile alphabets were displayed by the HapTip device that we evaluated in terms of position accuracy. Future work could be carried out with other devices to study the influence of the mean position accuracy on the alphabets' recognition rate. In the Morse alphabet, the design of letters was based on temporal signals, while TwoDashes used symbolic figures, and Unistrokes are a simplified representation of the Roman alphabet. Our tactile alphabets were evaluated in terms of recognition rate and learning. Results showed recognition rates from $80 \%$ to $97 \%$ after 5 sessions. As expected, our tactile version of the Morse code is the most efficient in terms of recognition rate. TwoDashes and mostly Unistrokes demonstrate the possibility to convey letters in a more intuitive and easier to learn way than Morse code. Besides, Unistrokes displayed the shortest association time. Taken together, our results pave the way to novel kinds of haptic communication using skin stretch.

\section{ACKNOWLEDGMENTS}

\section{REFERENCES}

[1] Barber, D., Reinerman-Jones, L., Matthews, G.: Toward a tactile language for human-robot interaction: two studies of tacton learning and performance. Human factors 57(3), 471-490 (2015)

[2] Basbaum, I.A.I., Kaneko, A., Shepherd, G.M., Westheimer, G., Senses, T., Reference, A.C., Gardner, E., Jon, H.: Tactile Sensory Control of Object Manipulation in Humans. Control 6, 67-86 (2008)

[3] Benali-Khoudja, M., Hafez, M., Sautour, A., Jumpertz, S.: Towards a new tactile language to communicate emotions. IEEE International Conference Mechatronics and Automation pp. 286-291 (2005)

[4] Brewster, S., Brown, L.M.: Tactons: Structured Tactile Messages for Non-Visual Information Display. In: Proc. of Australasian User Interface Conference. vol. 28, pp. 15-23 (2001)

[5] Bright, W.G., Engstrom, E.: Wireless Mobile Phone with Morse Code and Related Capabilities (2002)

[6] Caporusso, N.: A wearable Malossi alphabet interface for deafblind people. In: Proc. of the working conference on Advanced visual interfaces. pp. 445-448 (2008)
[7] Castellucci, S.J., MacKenzie, I.S.: Graffiti vs. Unistrokes: An Empirical Comparison. In: Proc. of Computer-Human Interaction. pp. 305-308 (2008)

[8] Chan, A., MacLean, K., McGrenere, J.: Learning and Identifying Haptic Icons under Workload. In: Symposium on Haptic Interfaces for Virtual Environment and Teleoperator Systems. pp. 432-439. Ieee (2005)

[9] Chan, A., MacLean, K., McGrenere, J.: Designing haptic icons to support collaborative turn-taking. International Journal of Human Computer Studies 66(5), 333-355 (2008)

[10] Chouvardas, V., a.N. Miliou, Hatalis, M.: Tactile displays: a short overview and recent developments. ICTA '05: Proceedings of Fifth International Conference on Technology and Automation (January), 246-251 (2005)

[11] Dinh, V.V., Phan, H.V., Tran, N.X., Ceruti, M.G., Ton, T.A., Duffy, L.: Wireless haptic glove for language and information transference (2010)

[12] Essock, E.a., Krebs, W.K., Prather, J.R.: Superior sensitivity for tactile stimuli oriented proximally-distally on the finger: Implications for mixed class 1 and class 2 anisotropies. Journal of Experimental Psychology: Human Perception and Performance 23(2), 515-527 (1997)

[13] Gaffary, Y., Marchal, M., Girard, A., Pellan, M., Peigne, B., Emily, M., Gosselin, F., Anatole, L.: Studying one and two-finger perception of tactile directional cues. In: EuroHaptics. pp. 1-10 (2016)

[14] Girard, A., Marchal, M., Gosselin, F., Chabrier, A., Louveau, F., Lécuyer, A.: HapTip: Displaying Haptic Shear Forces at the Fingertips for Multi-Finger Interaction in Virtual Environments. Frontiers in ICT 3, 6 (2016)

[15] Gleeson, B., Horschel, S., Provancher, W.: Design of a FingertipMounted Tactile Display with Tangential Skin Displacement Feedback. IEEE Transactions on Haptics 3(4), 297-301 (2010)

[16] Gollner, U., Bieling, T., Joost, G.: Mobile Lorm Glove: introducing a communication device for deaf-blind people. In: Proc. of the nternational Conference on Tangible, Embedded and Embodied Interaction. vol. 1, p. 127 (2012)

[17] Gwilliam, L.T., Doxon, A.J., Provancher, W.R.: Haptic matching of directional force and skin stretch feedback cues. World Haptics Conference pp. 19-24 (2013)

[18] Hoggan, E., Brewster, S.: New Parameters for Tacton Design. In: CHI Extended Abstracts on Human Factors in Computing Systems. pp. 2417-2422 (2007)

[19] Johansson, B.Y.R.S., Vallbo, A.B.: Tactile Sensibility in the Human Hand: Relative and Absolute Densities of Four Types of Mechanoreceptive Units in Glabrous Skin. Journal of Physiology 286, 283-300 (1979)

[20] Johnson, K.O.: The roles and functions of cutaneous mechanoreceptors. Current Opinion in Neurobiology 11(4), 455-461 (2001)

[21] Levine, S., Gauger, J., Bowers, L., Khan, K.: A comparison of Mouthstick and Morse code text inputs. Augmentative and Alternative Communication 2(2), 51-55 (2009)

[22] Liao, Y.C., Chen, Y.L., Lo, J.Y., Liang, R.H., Chan, L., Chen, B.Y.: EdgeVib: Effective Alphanumeric Character Output Using a WristWorn Tactile Display. In: Annual Symposium on User Interface Software and Technology. pp. 595-601 (2016)

[23] Luo, C.H., Shih, C.H.: Adaptive Morse-coded single-switch communication system for the disabled. International Journal of BioMedical Computing 41(2), 99-106 (1996) 
[24] MacKenzie, I.S., Zhang, S.X.: The immediate usability of Graffiti. In: Proc. of the conference on Graphics interface. pp. 129-137 (1997)

[25] Mandai, O., Guerrien, A., Sockeel, P., Dujardin, K., Leconte, P.: REM sleep modifications following a Morse code learning session in humans. Physiology and Behavior 46(4), 639-642 (1989)

[26] Nicolau, H., Guerreiro, J., Guerreiro, T., Carriço, L.: UbiBraille: designing and evaluating a vibrotactile Braille-reading device. In: Proc. of ACM SIGACCESS Conference on Computers and Accessibility. pp. 1-8 (2013)

[27] Pasquero, J., Hayward, V.: STReSS : A Practical Tactile Display System with One Millimeter Spatial Resolution and 700 $\mathrm{Hz}$ Refresh Rate. Proc Eurohaptics 2003(July), 94-110 (2003), http://cim.mcgill.ca/ haptic/pub/JP-VH-EH-03.pdf

[28] Ploch, C.J., Hwa, J., Wendy, B., Cutkosky, M.: Haptic Skin Stretch on a Steering Wheel for Displaying Preview Information in Autonomous Cars. In: Proc. of the IEEE/RSJ International Conference on Intelligent Robots and Systems. pp. 60-65 (2016)

[29] Qian, H., Kuber, R., Sears, A.: Towards identifying distinguishable tactons for use with mobile devices. Proc. of the International ACM SIGACCESS Conference on Computers and Accessibility p. 257 (2009)

[30] Rantala, J., Raisamo, R., Lylykangas, J., Surakka, V., Raisamo, J., Salminen, K., Pakkanen, T., Hippula, A.: Methods for presenting braille characters on a mobile device with a touchscreen and tactile feedback. IEEE Transactions on Haptics 2(1), 28-39 (2009)

[31] Rovers, L., Essen, H.V.: Design and evaluation of Hapticons for enriched Instant Messaging (2004)

[32] de Ruiter, J.: Application of Morse Code or Other Encoding Method to Instant Messaging and Incoming Calls on Mobile Devices. Patent US20130084902 p. 4 (2013)

[33] Sadato, N.: How the Blind "See" Braille: Lessons From Functional Magnetic Resonance Imaging. The Neuroscientist 11(6), 577-582 (2005) http://nro.sagepub.com/cgi/doi/10.1177/107385840527

[34] Sears, A.B.: A comparison of hypnotic and waking learning of the international morse code. Journal of Clinical and Experimental Hypnosis 3(4), 215-221 (1955)

[35] Sterr, A., Green, L., Elbert, T.: Blind Braille readers mislocate tactile stimuli. Biological Psychology 63(2), 117-127 (2003)

[36] Tan, H.Z., Durlach, N.I., Rabinowitz, W.M., Reed, C.M., Santos, J.R.: Reception of Morse code through motional, vibrotactile and auditory stimulation - Tan.pdf. Perception \& psychophysics 59(7), 1004-1017 (1997)

[37] Ternes, D., MacLean, K.E.: Designing large sets of Haptic icons with rhythm. Lecture Notes in Computer Science (including subseries Lecture Notes in Artificial Intelligence and Lecture Notes in Bioinformatics) 5024 LNCS, 199-208 (2008)

[38] Van Boven, R.W., Hamilton, R.H., Kauffman, T., Keenan, J.P., Pascual-Leone, a.: Tactile spatial resolution in blind braille readers. Neurology 54(12), 2230-2236 (2000)

[39] Yang, C.H., Huang, H.C., Chuang, L.Y., Yang, C.H.: A mobile communication aid system for persons with physical disabilities. Mathematical and Computer Modelling 47(3-4), 318-327 (2008)

\section{ABOUT THE AUTHORS}

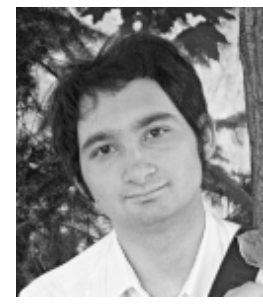

Yoren Gaffary is a Post-doctoral fellow at Inria on tactile perception and interaction design. His research interests are in affective computing, haptic and augmented reality. He received a master's degree in Information, Learning and Cognition at Paris South University. His Ph.D. thesis at University of Paris-Sud, France, concerned affective computing using mediated touch with robotic devices coupled with virtual humans.

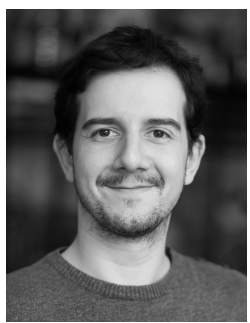

Ferran Argelaguet is a Research Scientist at the Hybrid research team, at Inria, the French National Institute for Research in Computer Science and Control, in Rennes, France. His research interests include 3D user interfaces, virtual reality and human perception. He received his PhD in Computer Science at the Polytechnic University of Catalonia in 2011. He is currently Review Editor of "Frontiers in Virtual Environments".

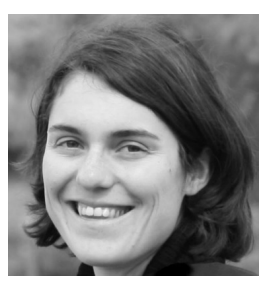

Maud Marchal is an Associate Professor in the Computer Science Department at INSA (Engineering school) in Rennes, France. Her main research interests include physically based simulation, haptic rendering, 3D interaction and virtual reality. She received her MS (2003) and PhD (2006) in Computer Science from the University Joseph Fourier in Grenoble, France.

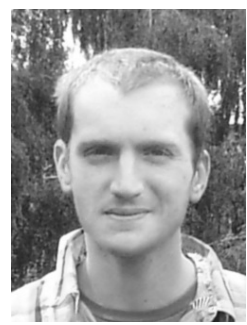

Adrien Girard is a Post-doctoral fellow at Inria on tactile stimulation at fingertips. His research interests are in haptic interaction and shared virtual environments. His Ph.D. thesis at University of Paris-Sud, France, concerned visio-haptic interactions for coordination and shared awareness in virtual and collaborative environments.

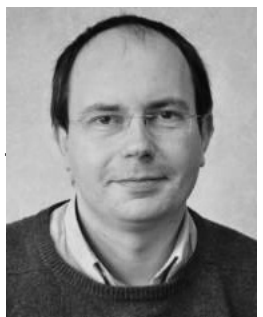

Florian Gosselin is a Research Engineer and project manager within the Interactive Robotics Laboratory, and the scientific officer of the Department of Ambient Intelligence and Interactive Systems at CEA-LIST in Gif-sur-Yvette, France. He received a Ph.D. in Mechanical Engineering from the University of Poitiers, France. His research interests include the electro-mechanical design of high performance haptic interfaces and collaborative robots as well as their integration in multimodal VR platforms and working environ-

ments.

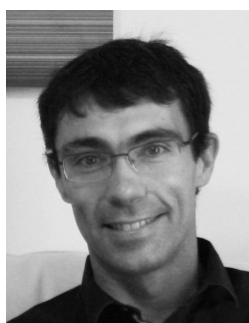

Mathieu Emily is an Associate Professor in Statistics and head of the Department of Statistics and Computer Sciences at Agrocampus Ouest (Engineering school) in Rennes. His research interests include biostatistics and spatial statistics. He received his MS (2003) and PhD (2006) in applied mathematics from the Institut National Polytechnique in Grenoble, France.

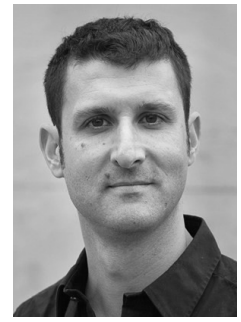

Anatole Lécuyer is Senior Researcher and head of Hybrid research team, at Inria Rennes, France. $\mathrm{He}$ is currently Associate Editor of "IEEE TVCG", "Frontiers in Virtual Environments" and "Presence" journals, and formerly of "ACM Transactions on Applied Perception" and "IJHCS". He was Program Chair of IEEE Virtual Reality Conference (2015-2016) and IEEE Symposium on 3D User Interfaces (2012-2013). 